Vietnam Journal of Mechanics, VAST, Vol.32, No. 3 (2010), pp. $191-198$

\title{
ON MODELLING AND SIMULATION OF A MANIPULATOR UNDER CONSIDERATION OF A JAMMED JOINT
}

\author{
Nguyen Quang Hoang \\ Hanoi University of Technology
}

\begin{abstract}
In this paper, the modelling of a jamming process of a joint during the operation of a manipulator is presented. Based on the kinematic property of a jamming process, a motion law of the jammed joint is chosen. By introducing a matrix coressponding to jammed joint, the equation of motion of the system is restructured without re-deriving. Some numerical simulations are carried out to illustrate the proposed algorithm.
\end{abstract}

\section{INTRODUCTION}

During the operation of a manipulator or of a mechanical system failures can be occurred. One of these failures is jamming of a joint. The joint can be locked due to a jammed brake, the stuck of gear transmittion,... As consequence, there will be no relative motion between the two links that are connected through the jammed joint, i.e., the corresponding links and the joint will be reduced to a single link. The length of the resultant single link can be calculated from the lengths of two original links, and the relative position of these links after jamming occurs. A jammed joint will result in the reduction of a DOF of the manipulators. The issue of fault detection and isolation for serial and parallel manipulators have been investigated recently $[2-6,10,14,15]$. However, the modelling of a jamming process for simulation has not been discussed yet. The investigation on kinematics and dynamics of a manipulator in case a joint getting stuck can be carried out by examining of each case of the jammed joint. The approach method requires time and calculating burden.

In this paper, the dynamic problem of a manipulator in case of a joint getting stuck is considered. If the jamming time and the jammed joint are known, the equation of motion of a manipulator can be determined by introduction of the so-called structure matrix. This paper is organized as follows: in section 2 the dynamics model in case of a jammed joint is described. Section 3 shows some results of numerical simulations.

\section{MODELLING OF A JAMMING PROCESS}

The dynamic equations of a robot manipulator can be derived by using Lagrange's theory $[1,7-9,11-13]$ as

$$
\mathbf{M}(\mathbf{q}) \ddot{\mathbf{q}}+\mathbf{C}(\mathbf{q}, \dot{\mathbf{q}}) \dot{\mathbf{q}}+\mathbf{D}(\mathbf{q}, \dot{\mathbf{q}}) \dot{\mathbf{q}}+\mathbf{g}(\mathbf{q})=\mathbf{u}
$$


where $\mathbf{q} \in \mathrm{R}^{n}$ is the joint position vector, $\mathbf{M}(\mathbf{q}) \in \mathrm{R}^{n \times n}$ is the symmetric positive definite inertia matrix, $\mathbf{C}(\mathbf{q}, \dot{\mathbf{q}}) \in \mathrm{R}^{n \times n}$ is the Coriolis and centripetal matrix, $\mathbf{g}(\mathbf{q}) \in \mathrm{R}^{n}$ is the gravitational torque vector, $\mathbf{D}(\mathbf{q}, \dot{\mathbf{q}}) \dot{\mathbf{q}}$ is damping moments, and $\mathbf{u} \in \mathrm{R}^{n}$ is the applied torque vector.

The jamming process can occur suddenly and it is difficult to describe. In this paper, it is assumed that $k^{t h}$ joint starts jamming at time $t=t_{1}$, after a time interval $\Delta t=t_{2}-t_{1}$ this joint is completely jammed. During this time the relative velocity decreases to zero $\dot{q}_{k} \rightarrow 0$ and the joint variable is blocked at $q_{k} \rightarrow q_{k}\left(t_{2}\right)$. Mathematically, the motion of the jammed joint can be described as follows:

If $t_{1} \leq t<t_{2}: \ddot{q}_{k}(t)=-\dot{q}_{k}\left(t_{1}\right) / \Delta t, \dot{q}_{k}(t)=\dot{q}_{k}+\ddot{q}_{k}\left(t-t_{1}\right)$.

If $t_{2} \leq t: \quad \ddot{q}_{k}(t)=0, \dot{q}_{k}(t)=0$.

Here, in the jamming process the joint acceleration is assumed to be constant. In order to simulate, the operation of the manipulator can be devided into three phases:

- Normal phase (without jam), $0<t<t_{1}$

$$
\ddot{\mathbf{q}}=\mathbf{M}^{-1}(\mathbf{q})[\mathbf{u}-\mathbf{h}(\mathbf{q}, \dot{\mathbf{q}})-\mathbf{d}],
$$

with $\mathbf{h}(\mathbf{q}, \dot{\mathbf{q}})=\mathbf{C}(\mathbf{q}, \dot{\mathbf{q}}) \dot{\mathbf{q}}+\mathbf{D}(\mathbf{q}, \dot{\mathbf{q}}) \dot{\mathbf{q}}+\mathbf{g}(\mathbf{q})+\mathbf{d}$.

- Jamming phase (joint $k$ get stuck), $t_{1} \leq t<t_{2}$

$$
\begin{aligned}
& \mathbf{M}_{a}=\mathbf{E}_{k}^{T} \mathbf{M} \mathbf{E}_{k}, \quad \mathbf{h}_{a}=\mathbf{E}_{k}^{T} \mathbf{h}+\mathbf{E}_{k}^{T} \mathbf{M} \mathbf{e}_{k} \ddot{q}_{k}, \quad \mathbf{u}_{a}=\mathbf{E}_{k}^{T} \mathbf{u}, \\
& \ddot{\mathbf{q}}_{a}=\mathbf{M}_{a}^{-1}(\mathbf{q})\left[\mathbf{u}_{a}-\mathbf{h}_{a}(\mathbf{q}, \dot{\mathbf{q}})\right], \ddot{\mathbf{q}}=\mathbf{E}_{k} \ddot{\mathbf{q}}_{a}+\mathbf{e}_{k} \ddot{q}_{k} .
\end{aligned}
$$

- Joint $\mathrm{k}$ is blocked, $t_{2}<t$

$$
\begin{aligned}
& \ddot{q}_{k}(t)=0, \quad \dot{q}_{k}(t)=0, \mathbf{M}_{a}=\mathbf{E}_{k}^{T} \mathbf{M} \mathbf{E}_{k}, \quad \mathbf{h}_{a}=\mathbf{E}_{k}^{T} \mathbf{h}, \quad \mathbf{u}_{a}=\mathbf{E}_{k}^{T} \mathbf{u}, \\
& \ddot{\mathbf{q}}_{a}=\mathbf{M}_{a}^{-1}(\mathbf{q})\left[\mathbf{u}_{a}-\mathbf{h}_{a}(\mathbf{q}, \dot{\mathbf{q}})\right], \quad \ddot{\mathbf{q}}=\mathbf{E}_{k} \ddot{\mathbf{q}}_{a}+\mathbf{e}_{k} \ddot{q}_{k},
\end{aligned}
$$

where $\mathbf{E}_{k} \in \mathrm{R}^{n \times(n-1)}$ is the so-called structrure matrix obtained by deleting $k^{\text {th }}$ colume of the identical matrix of zise $n \times n$, and $\mathbf{e}_{k}$ is a $n \times 1$-vector with $e_{k}(k)=1, e_{k}(i)=0$, $(i \neq k)$.

Noting that when the joint $k$ gets stuck the degree of freedom of the manipulator is reduced by one. Hence, the inertia matrix $\mathbf{M}_{a}$, vectors $\mathbf{h}_{a}$, and $\mathbf{u}_{a}$ have a zise of $(n-1)$, e.g. $\mathbf{M}_{a} \in \mathrm{R}^{(n-1) \times(n-1)}, \mathbf{h}_{a} \in \mathrm{R}^{(n-1) \times 1}, \mathbf{u}_{a} \in \mathrm{R}^{(n-1) \times 1}$. Moreover, the matrix $\mathbf{M}_{a}$ is a symmetric positive definite one, because this is the inertia matrix of a mechanical system obtained by rigidening one joint of the original system. The value of $k \in(1, . ., n)$, jamming time moment $t_{1}>0$, and jamming duration $\Delta t=t_{2}-t_{1}>0$ can be given by a random number.

\section{NUMERICAL SIMULATION}

In this section, some simulations in universal software Matlab is implemented to illustrate the proposed algorithm. In this simulation, a 3-DOF planar manipulator moving in the vertical plane is considered. The $i^{t h}$ link has a length of $L_{i}$, a mass of $m_{i}$, and the inertia moment respect to $\mathrm{C}_{i}$ is $J_{C i}\left(\mathrm{C}_{i}\right.$ is a center of mass). The distance from $\mathrm{C}_{i}$ to the joint connected to $(i-1)^{t h} \operatorname{link}$ is $a_{i}$. The torque of motor fixed on link $(i-1)$ acts on link $i$ 


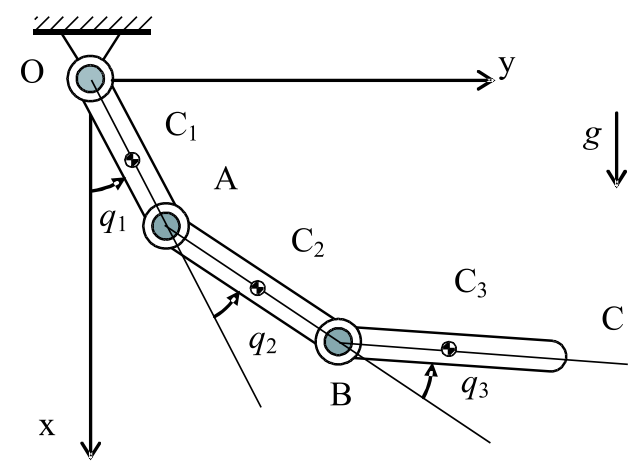

Fig. 1. A 3-DOF planar manipulator

is $u_{i}(i=1, \ldots, 3)$. The model of the manipulator and its parameters are shown in Fig. 1 and Table 1.

Let $q^{T}=\left[q_{1}, q_{2}, q_{3}\right]$ be the generalized coordinates of the manipulator, $q_{1}$ is the angle of link 1, bar OA; $q_{2}$ is the relative angle of link 2 respective to link 1 ; and $q_{3}$ is the relative angle of link 3 respective to link 2 . The kinetic and potential energy of the system are obtained as follows:

$$
\begin{aligned}
T=\frac{1}{2} & J_{C_{1}}+m_{1} a_{1}^{2}+J_{C_{2}}+m_{2} a_{2}^{2}+m_{2} l_{1}^{2}+2 m_{2} l_{1} a_{2} \cos q_{2}+2 m_{2} l_{2} a_{3} \cos q_{3} \\
& \left.+J_{C_{3}}+m_{3} a_{3}^{2}+m_{3} l_{1}^{2}+m_{3} l_{2}^{2}+2 m_{3} l_{1} a_{3} \cos \left(q_{2}+q_{3}\right)+2 m_{3} l_{1} l_{2} \cos q_{2}\right] \dot{q}_{1}^{2} \\
& +\left[J_{C_{2}}+m_{2} a_{2}^{2}+m_{2} l_{1} a_{2} \cos q_{2}+J_{C_{3}}+m_{3} a_{3}^{2}+m_{3} l_{2}^{2}\right. \\
& \left.+m_{3} l_{1} a_{3} \cos \left(q_{2}+q_{3}\right)+m_{3} l_{1} l_{2} \cos q_{2}+2 m_{3} l_{2} a_{3} \cos q_{3}\right] \dot{q}_{1} \dot{q}_{2} \\
& +\left[J_{C_{3}}+m_{3} a_{3}^{2}+m_{3} l_{2} a_{3} \cos q_{3}+m_{3} l_{1} a_{3} \cos \left(q_{2}+q_{3}\right)\right] \dot{q}_{1} \dot{q}_{3} \\
& \frac{1}{2}\left(J_{C_{2}}+m_{2} a_{2}^{2}+J_{C_{3}}+m_{3} a_{3}^{2}+m_{3} l_{2}^{2}+2 m_{3} l_{2} a_{3} \cos q_{3}\right) \dot{q}_{2}^{2} \\
& +\left(J_{C_{3}}+m_{3} a_{3}^{2}+m_{3} l_{2} a_{3} \cos q_{3}\right) \dot{q}_{2} \dot{q}_{3}+\frac{1}{2}\left(J_{C_{3}}+m_{3} a_{3}^{2}\right) \dot{q}_{3}^{2} \\
& \quad-\sum_{i=1}^{3} m_{i} g x_{C i}=-\left\{m_{1} g a_{1} \cos q_{1}+m_{2} g\left[l_{1} \cos q_{1}+a_{2} \cos \left(q_{1}+q_{2}\right)\right]+\right. \\
& \left.\quad+m_{3} g\left[l_{1} \cos q_{1}+l_{2} \cos \left(q_{1}+q_{2}\right)+a_{3} \cos \left(q_{1}+q_{2}+q_{3}\right)\right]\right\} ;
\end{aligned}
$$

The applied torques of motors acting on links and damping at each joint are given by the principle of virtual work:

$$
Q_{1}=-b_{1} \dot{q}_{1}+u_{1}, Q_{2}=-b_{2} \dot{q}_{2}+u_{2}, Q_{3}=-b_{3} \dot{q}_{3}+u_{3}
$$

Applying the Lagrangian equation of the second kind one obtains the equation of motion in matrix form as (1). The differential equation of motion (1) will be implemented in Matlab simulink for simulation as in Fig. 2.

In order to check the simulation results, the case of the free motion of the manipulator is investigated. It is clearly that there are no torques applying on the manipulator, 
Table 1. Some parameters of the manipulator

\begin{tabular}{|c|c|c|c|}
\hline Link & 1 & 2 & 3 \\
\hline$m[\mathrm{~kg}]$ & 9.00 & 7.00 & 5.00 \\
\hline$J_{C}\left[\mathrm{kgm}^{2}\right]$ & 1.50 & 1.00 & 0.60 \\
\hline$L[\mathrm{~m}]$ & 0.80 & 0.70 & 0.60 \\
\hline$a[\mathrm{~m}]$ & 0.40 & 0.35 & 0.30 \\
\hline
\end{tabular}

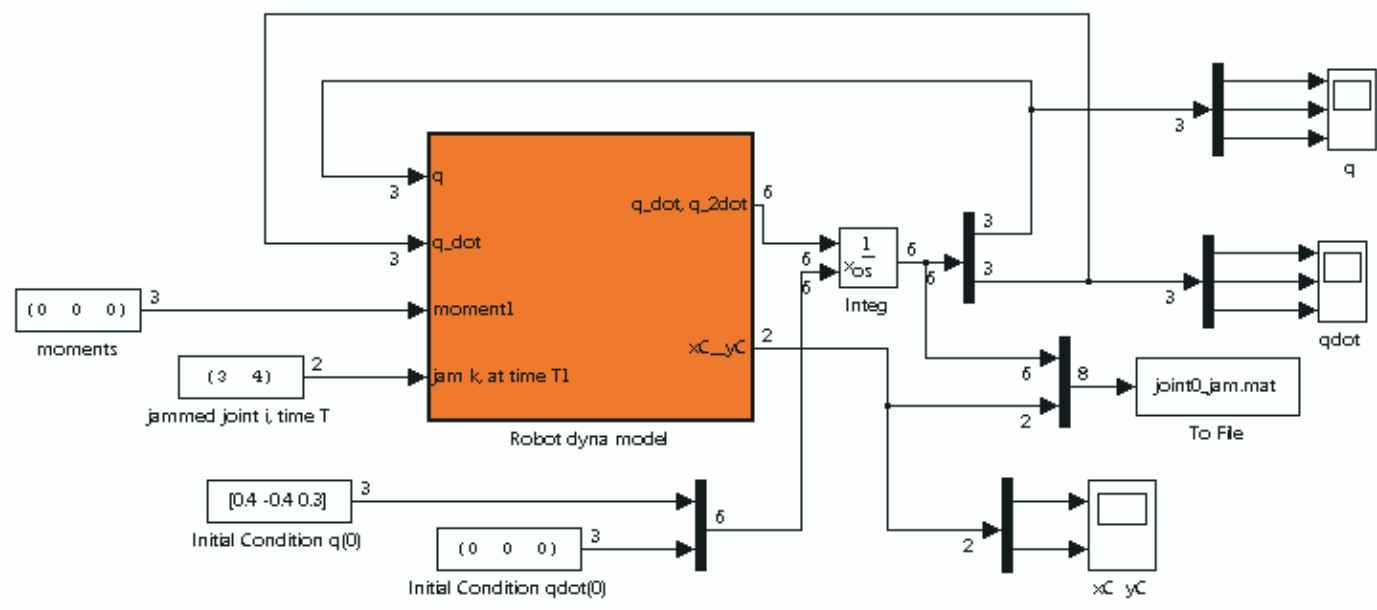

Fig. 2. The simulink diagram

so the manipulator will move to stable equilibrium $\mathbf{q}_{e}=\left[\begin{array}{lll}0 & 0 & 0\end{array}\right]^{T}$ from any arbitrary initial position due to gravitational forces and damping at each joint. In simulations the coordinates of the center of mass is also given to check the reliability of the presented approach.

Three numerical simulations are carried out: In the first case, there are no fault (no jammed joint), the second case joint 1 gets stuck at time $t_{1}=2 \mathrm{~s}$. And in the last simulation, joint 2 gets stuck at time $t_{1}=0.5 \mathrm{~s}$. The simulation results are shown in Fig. 3 to Fig. 6.

Fig. 3 present the simulation results of the first case: the manipulator moves to the equilibrium $\mathbf{q}_{e}=\left[\begin{array}{lll}0 & 0 & 0\end{array}\right]^{T}$ after about $15 \mathrm{~s}$. In this case, the center of mass is located at 

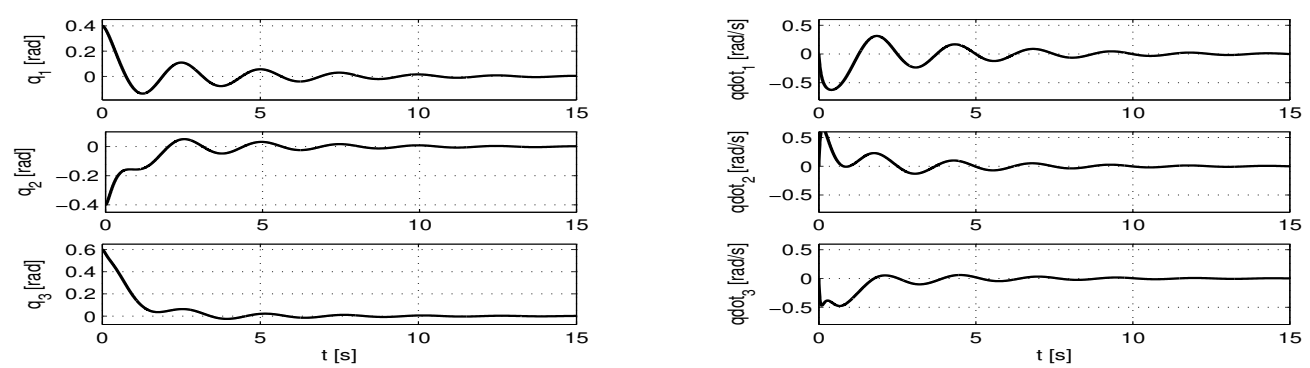

Fig. 3. Time history of generalized coordinates (1. case, no jammed joint)
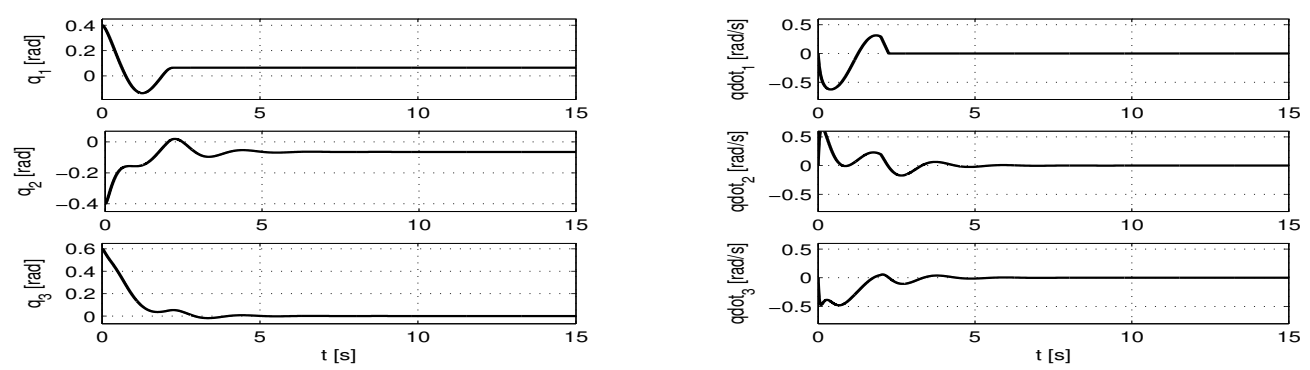

Fig. 4. Time history of generalized coordinates (2. case, joint 1 gets stuck)
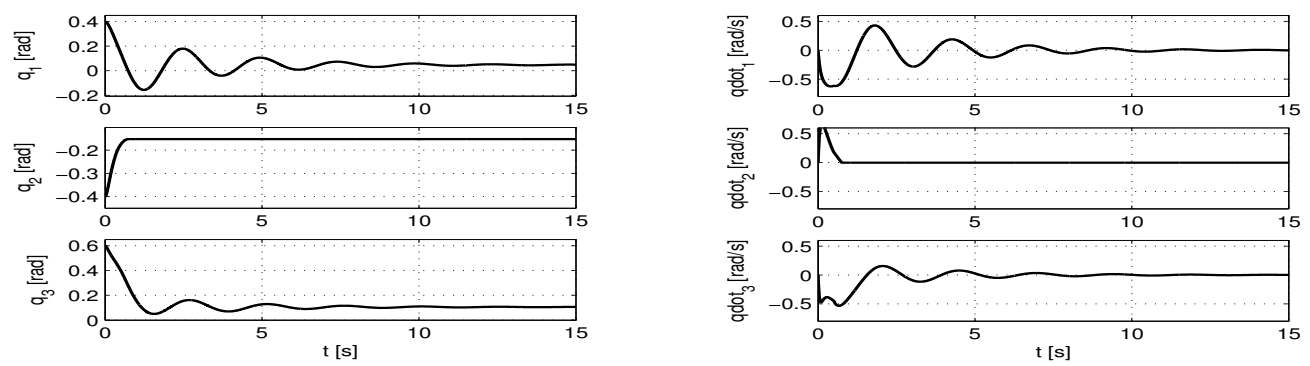

Fig. 5. Time history of generalized coordinates (3. case, joint 2 gets stuck)

the lowest position, and $\mathrm{y}_{C}=0$ (Fig. 6). The results of the second case are shown in Fig. 4. The joints 1 gets jamming at the time $t_{1}=2 \mathrm{~s}$, after that $q_{1}$ becomes a constant, and the system moves to the equilibrium $\mathbf{q}_{e}=[0.0645,-0.0645,0.0]^{T}$ after about $10 \mathrm{~s}$. The results show that in the equilibrium $q_{1}=-q_{2}$, and $q_{3}=0.0$.

The results of the last case are shown in Fig. 5. Joints 2 gets jamming at time $t_{1}=0.5 \mathrm{~s}$, after that $q_{2}$ becomes a constant, and the system moves to the equilibrium $\mathbf{q}_{e}=\left[\begin{array}{lll}0.0473-0.1525 & 0.1051\end{array}\right]^{T}$ after about $15 \mathrm{~s}$. The center of mass in the equilibrium lies on the $x$ axis, $y_{C}=0$. 

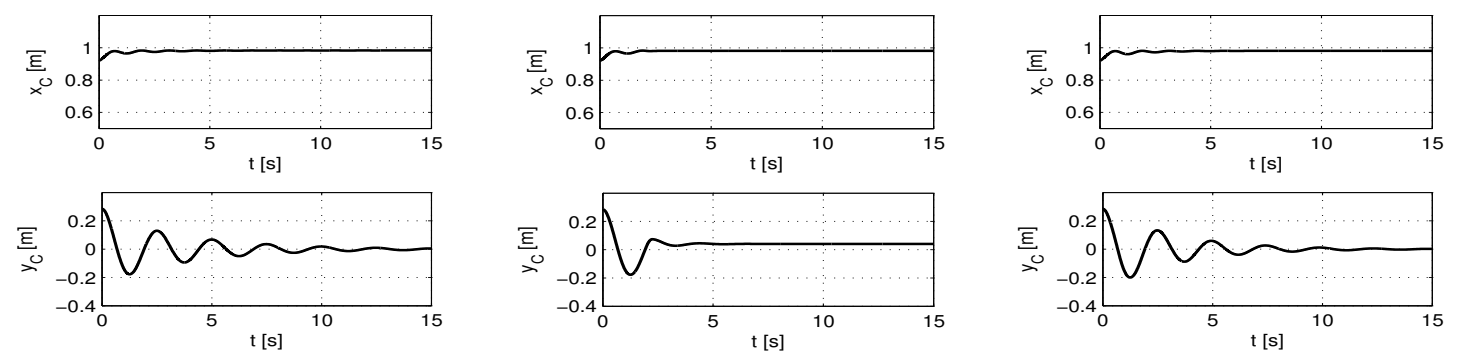

Case 2

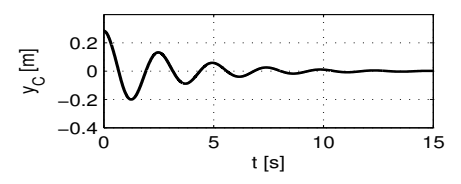

Case 3

Fig. 6. Coordinates of the center of mass

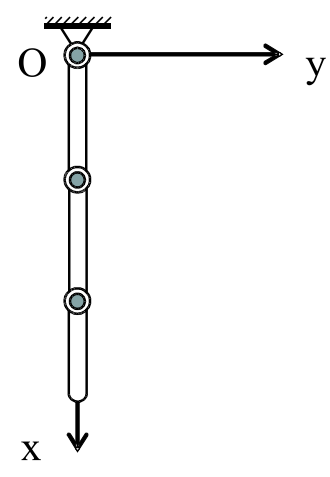

Case 1: No fault $\mathbf{q}_{e}=[0,0,0]^{T}$

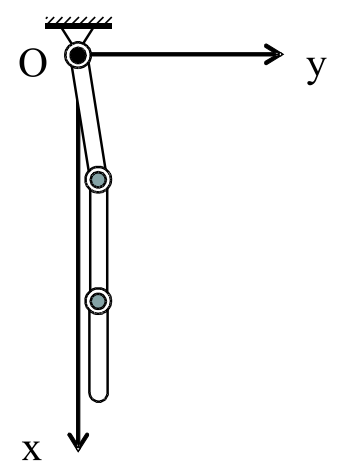

Case 2: Jam at joint 1 $\mathbf{q}_{e}=[0.0645,-0.0645,0.0]^{T}$

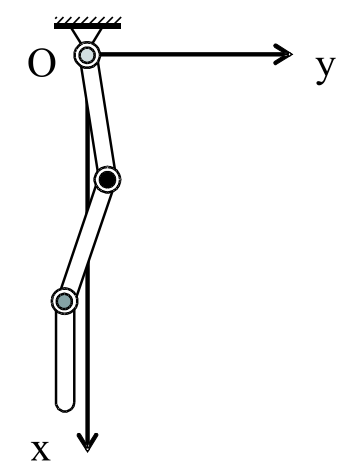

Case 3: Jam at joint 2 $\mathbf{q}_{e}=[0.0473,-0.1525,0.1051]^{T}$

Fig. 7. Some manipulator configurations in equilibrium

Fig. 7 shows the configurations of the manipulator in equilibrium in case of nofault, jamming at joint 1 , and jamming at joint 2. Visually, these configurations are close to reality, because the center of mass is always located at the lowest position due to gravitation.

\section{CONSLUSION}

In reality, when a joint is completely jammed, the manipulator structure and the number of degrees of freedom will be changed. This leads to change the forward kinematics. Thus we have to solve the problem for each case of jam of joints. This work requires time and calculating burden. The presented algorithm in this study has overcome these difficulties. By choosing a motion law of the jammed joint and introducing the so-called restructured matrix, the equation of motion of the system is restructured without rederiving. The effectiveness of the proposed method is demonstrated by means of numerical experiments. 


\section{APPENDIX}

- Kinetic energy of the maniupulator: $T=\frac{1}{2} J_{O_{1}} \omega_{1}^{2}+\frac{1}{2} \sum_{i=2}^{3}\left(m_{i} v_{C i}^{2}+J_{C i} \omega_{i}^{2}\right)$

Link 1: $J_{O_{1}}=J_{C 1}+m_{1} a_{1}^{2}, \quad \omega_{1}=\dot{q}_{1}$.

Link 2: $x_{C 2}=l_{1} \cos q_{1}+a_{2} \cos \left(q_{1}+q_{2}\right), \quad \dot{x}_{C 2}=-l_{1} \dot{q}_{1} \sin q_{1}-a_{2}\left(\dot{q}_{1}+\dot{q}_{2}\right.$

$y_{C 2}=l_{1} \sin q_{1}+a_{2} \sin \left(q_{1}+q_{2}\right), \quad \dot{y}_{C 2}=l_{1} \dot{q}_{1} \cos q_{1}+a_{2}\left(\dot{q}_{1}+\dot{q}_{2}\right) \cos \left(q_{1}+q_{2}\right)$,

$\omega_{2}=\left(\dot{q}_{1}+\dot{q}_{2}\right), \quad v_{C 2}^{2}=\dot{x}_{C 2}^{2}+\dot{y}_{C 2}^{2}=l_{1}^{2} \dot{q}_{1}^{2}+a_{2}^{2}\left(\dot{q}_{1}+\dot{q}_{2}\right)^{2}+2 l_{1} a_{2} \dot{q}_{1}\left(\dot{q}_{1}+\dot{q}_{2}\right) \cos q_{2}$.

Link 3: $x_{C 3}=l_{1} \cos q_{1}+l_{2} \cos \left(q_{1}+q_{2}\right)+a_{3} \cos \left(q_{1}+q_{2}+q_{3}\right)$,

$y_{C 3}=l_{1} \sin q_{1}+l_{2} \sin \left(q_{1}+q_{2}\right)+a_{3} \sin \left(q_{1}+q_{2}+q_{3}\right)$,

$\dot{x}_{C 3}=-l_{1} \dot{q}_{1} \sin q_{1}-l_{2}\left(\dot{q}_{1}+\dot{q}_{2}\right) \sin \left(q_{1}+q_{2}\right)-a_{3}\left(\dot{q}_{1}+\dot{q}_{2}+\dot{q}_{3}\right) \sin \left(q_{1}+q_{2}+q_{3}\right)$,

$\dot{y}_{C 3}=l_{1} \dot{q}_{1} \cos q_{1}+l_{2}\left(\dot{q}_{1}+\dot{q}_{2}\right) \cos \left(q_{1}+q_{2}\right)+a_{3}\left(\dot{q}_{1}+\dot{q}_{2}+\dot{q}_{3}\right) \cos \left(q_{1}+q_{2}+q_{3}\right)$,

$\omega_{2}=\left(\dot{q}_{1}+\dot{q}_{2}+\dot{q}_{3}\right), \quad v_{C 3}^{2}=\dot{x}_{C 3}^{2}+\dot{y}_{C 3}^{2}$.

- The inertia matrix $\mathbf{M}(\mathbf{q}) \in \mathrm{R}^{n \times n}$ :

$M_{11}=2 m_{3} l_{1} l_{2} \cos q_{2}+2 m_{3} l_{1} a_{3} \cos \left(q_{2}+q_{3}\right)+2 m_{2} l_{1} a_{2} \cos q_{2}+J_{C 2}+m_{2} a_{2}^{2}+$ $+m_{1} a_{1}^{2}+2 m_{3} l_{2} a_{3} \cos q_{3}+J_{C 1}+m_{3} l_{2}^{2}+J_{C 3}+m_{3} a_{3}^{2}+m_{3} l_{1}^{2}+m_{2} l_{1}^{2}$,

$M_{12}=J_{C 2}+m_{3} l_{1} a_{3} \cos \left(q_{2}+q_{3}\right)+m_{3} a_{3}^{2}+J_{C 3}+m_{2} l_{1} a_{2} \cos q_{2}+$ $+2 m_{3} l_{2} a_{3} \cos q_{3}+m_{2} a_{2}^{2}+m_{3} L_{2}^{2}+m_{3} l_{1} l_{2} \cos q_{2}$,

$M_{13}=m_{3} l_{2} a_{3} \cos q_{3}+J_{C 3}+m_{3} l_{1} a_{3} \cos \left(q_{2}+q_{3}\right)+m_{3} a_{3}^{2}$,

$M_{21}=M_{12} \quad M_{23}=J_{C 3}+m_{3} l_{2} a_{3} \cos q_{3}+m_{3} a_{3}^{2}$,

$M_{22}=J_{C 3}+2 m_{3} l_{2} a_{3} \cos q_{3}+J_{C 2}+m_{2} a_{2}^{2}+m_{3} a_{3}^{2}+m_{3} l_{2}^{2}$,

$M_{31}=M_{13}, \quad M_{32}=M_{23}, \quad M_{33}=m_{3} a_{3}^{2}+J_{C 3}$

- The Coriolis and centripetal matrix $\mathbf{C}(\mathbf{q}, \dot{\mathbf{q}}) \in \mathrm{R}^{n \times n}$ :

$C_{11}=-m_{3} l_{1} a_{3} \dot{q}_{2} \sin \left(q_{2}+q_{3}\right)-m_{3} l_{1} l_{2} \dot{q}_{2} \sin q_{2}-m_{2} l_{1} a_{2} \dot{q}_{2} \sin q_{2}$

$-m_{3} l_{1} a_{3} \dot{q}_{3} \sin \left(q_{2}+q_{3}\right)-m_{3} l_{2} a_{3} \dot{q}_{3} \sin q_{3}$,

$C_{12}=-m_{2} l_{1} a_{2} \dot{q}_{2} \sin q_{2}-m_{3} l_{1} l_{2} \dot{q}_{2} \sin q_{2}-m_{3} l_{1} a_{3} \dot{q}_{2} \sin \left(q_{2}+q_{3}\right)-m_{3} l_{1} a_{3} \dot{q}_{3} \sin \left(q_{2}+q_{3}\right)$

$-m_{3} L_{2} a_{3} \dot{q}_{3} \sin q_{3}-m_{3} l_{1} a_{3} \dot{q}_{1} \sin \left(q_{2}+q_{3}\right)-m_{3} l_{1} l_{2} \dot{q}_{1} \sin q_{2}-m_{2} l_{1} a_{2} \dot{q}_{1} \sin q_{2}$,

$C_{13}=-m_{3} a_{3}\left[l_{1} \dot{q}_{2} \sin \left(q_{2}+q_{3}\right)+l_{2} \dot{q}_{3} \sin q_{3}+l_{1} \dot{q}_{3} \sin \left(q_{2}+q_{3}\right)\right.$ $\left.+l_{1} \dot{q}_{1} \sin \left(q_{2}+q_{3}\right)+l_{2} \dot{q}_{1} \sin q_{3}+l_{2} \dot{q}_{2} \sin q_{3}\right]$

$C_{21}=-m_{3} L_{2} a_{3} \sin \left(q_{3}\right) \dot{q}_{3}+m_{3} L_{1} a_{3} \sin \left(q_{2}+q_{3}\right) \dot{q}_{1}+\dot{q}_{1} m_{3} L_{1} L_{2} \sin \left(q_{2}\right)+\dot{q}_{1} m_{2} L_{1} a_{2} \sin \left(q_{2}\right)$,

$C_{22}=-m_{3} L_{2} a_{3} \sin \left(q_{3}\right) \dot{q}_{3}, \quad C_{23}=-m_{3} L_{2} a_{3} \sin \left(q_{3}\right)\left(\dot{q}_{3}+\dot{q}_{1}+\dot{q}_{2}\right)$,

$C_{31}=m_{3} a_{3}\left(\dot{q}_{1} L_{1} \sin \left(q_{2}+q_{3}\right)+\dot{q}_{1} L_{2} \sin \left(q_{3}\right)+L_{2} \sin \left(q_{3}\right) \dot{q}_{2}\right)$,

$C_{32}=m_{3} L_{2} a_{3} \sin \left(q_{3}\right)\left(\dot{q}_{1}+\dot{q}_{2}\right), \quad C_{33}=0$.

- The gravitational torque vector $\mathbf{g}(\mathbf{q}) \in \mathrm{R}^{n}$ :

$$
\mathbf{g}(\mathbf{q})=\left[\begin{array}{c}
m_{1} g\left(a_{1} \sin q_{1}\right)+m_{2} g\left[l_{1} \sin q_{1}+a_{2} \sin \left(q_{1}+q_{2}\right)\right]+\ldots \\
m_{3} g\left[l_{1} \sin q_{1}+l_{2} \sin \left(q_{1}+q_{2}\right)+a_{3} \sin \left(q_{1}+q_{2}+q_{3}\right)\right. \\
m_{2} g a_{2} \sin \left(q_{1}+q_{2}\right)+m_{3} g\left[l_{2} \sin \left(q_{1}+q_{2}\right)+a_{3} \sin \left(q_{1}+q_{2}+q_{3}\right)\right] \\
m_{3} g a_{3} \sin \left(q_{1}+q_{2}+q_{3}\right)
\end{array}\right]
$$

- The applied torque vector $\mathbf{u} \in \mathrm{R}^{n}: \mathbf{u}=\left[\begin{array}{lll}u_{1}, & u_{2}, & u_{3}\end{array}\right]^{T}$.

\section{REFERENCES}

[1] J. Angeles, Fundamentals of robotics Mechanical Systems (2 ${ }^{\text {nd }}$ Edition), Springer Verlag, New York (2003). 
[2] W. E. Dixon, I.D. Walker, D.M. Dawson and J.P. Hartranft, Fault Detection for Robot Manipulators with Parametric Uncertainty: A Prediction-Error-Based Approach, IEEE Transactions on robotics and automation 16 (6) (2006) 689-699.

[3] M. Hassan and L. Notash, Design modification of parallel manipulators for optimum fault tolerance to joint jam, Mechanism and Machine Theory 40 (2005) 559-577.

[4] M. Hassan and L. Notash, Optimizing fault tolerance to joint jam in the design of parallel robot manipulators, Mechanism and Machine Theory 42 (2007) 1401-1417.

[5] Nguyen Q. Hoang, Nguyen V. Khang and Tran Hoang Nam, On kinematic inverse of redundant manipulators under consideration of jammed joint, Proceedings of the National Conference on Mechnics, 30 years Institute of Mechanics and Journal of Mechanics (2009) 255-263 (in Vietnamese).

[6] Nguyen Q. Hoang, Nguyen V. Khang, On kinematic inverse and control of redundant manipulators under consideration of jammed joint, Proceedings of the $1^{\text {st }}$ Int. Symp. on Robotics and Mechatronics (2009) 201-207.

[7] Nguyen Van Khang, Dynamics of Multibody Systems, Technical and Scienctific Publisher, Hanoi (2007) (in Vietnamese).

[8] R.M. Murray, Z. Li and S.S. Sastry, A Mathematical Introduction to Robotic Manipulation, CRC Press, Boca Raton, Fla. (1994).

[9] Y. Nakamura, Advanced Robotics/Redundancy and Optimization, Addison-Wesley Publishing Company, Reading (1991).

[10] L. Notash and L. Huang, On the design of fault tolerant parallel manipulators, Mechanism and Machine Theory 38 (2003) 85-101.

[11] Nguyen Thien Phuc, Industrial Robotics, Technical and Scienctific Publisher, Hanoi (2002) (in Vietnamese).

[12] L. Sciavicco and B. Siciliano, Modelling and Control of Robot Manipulators (2 ${ }^{\text {nd }}$ Edition), Springer-Verlag, London, UK (2000).

[13] M. W. Spong, S. Hutchinson and M. Vidyasagar, Robot Modeling and Control, John Wiley \& Sons, New York (2006).

[14] R. Tinos, M.H. Terra and M. Bergerman, A fault tolerance framework for cooperative robotic manipulators, Control Engineering Practice 15 (2007) 615-625.

[15] M.L. Visinsky, J.R. Cavallaro and I. D. Walker, Robotic fault detection and fault tolerance: A survey, Reliability Engineering and System Safety 46 (1994) 139-158.

Received May 07, 2010

\section{VỀ VIỆC MÔ HİNH HÓA VÀ MÔ PHỎNG CHUYỂN ĐộNG CỦA TAY MÁY CÓ CHÚ Ý ĐẾN SỰ CỐ KẸT KHỚP}

Trong bài báo này, việc mô hình hóa quá trình kẹt của khớp trong khi tay máy vận hành được trình bày. Trên cơ sở phân tích tính chất động học của quá trình kẹt, một luật chuyển động của khớp kẹt được chọn để mô tả động học quá trình kẹt. Bằng cách đưa vào ma trận cấu trúc tương ứng với khớp kẹt, phương trình vi phân chuyển động của hệ được cấu trúc lại mà không cần phải thiết lập lại. Từ đó giảm được thời gian phân tích động học và động lực học của hệ khi cấu trúc thay đổi do khớp bị kẹt. Các mô phỏng số được thực hiện bằng phần mềm đa năng Matlab để minh họa cho thuật toán. Kết quả mô phỏng đó khẳng định tính đúng đắn và phù hợp của phương pháp tiếp cận trong bài báo. 